\title{
Identification of High-Affinity Small Molecule Targeting IDH2 for the Clinical Treatment of Acute Myeloid Leukemia
}

\author{
Jajoriya Sweta ${ }^{1}$, Ravina Khandelwal ${ }^{1}$, Sivaraj Srinitha ${ }^{1}$, Rashi Pancholi ${ }^{1}$, \\ Ritu Adhikary ${ }^{1}$, Meer Asif Ali ${ }^{1}$, Anuraj Nayarisseri1 ${ }^{1,2,3 *}$, Sugunakar Vuree ${ }^{4}$, \\ Sanjeev Kumar Singh ${ }^{3 *}$
}

\begin{abstract}
Acute myeloid leukemia (AML) is symbolized by an increase in the number of myeloid cells in the bone marrow and an arrest in their maturation, frequently resulting in hematopoietic insufficiency (granulocytopenia, thrombocytopenia, or anemia) with or without leukocytosis either by a predominance of immature forms or a loss of normal hematopoiesis. IDH2 gene encodes for isocitrate dehydrogenase enzyme which is involved in the TCA cycle domino effect and converts isocitrate to alpha-ketoglutarate. In the U.S, the annual incidence of AML progressively increases with age to a peak of 12.6 per 100,000 adults of 65 years or older. Mutations in isocitrate dehydrogenase 2 (arginine 132) have been demonstrated to be recurrent gene alterations in acute myeloid leukemia (AML) by forming 2-Hydroxy alpha ketoglutarate which, instead of participating in TCA cycle, accumulates to form AML. The current study approaches by molecular docking and virtual screening to elucidate inhibitor with superior affinity against $I D H 2$ and achieve a pharmacological profile. To obtain the best established drug Molegro Virtual Docker algorithm was executed. The compound AG-221 (Pub CID 71299339) having the high affinity score was subjected to similarity search to retrieve the drugs with similar properties. The virtual screened compound SCHEMBL16391748 (PubChem CID-117816179) shows high affinity for the protein. Comparative study and ADMET study for both the above compounds resulted in equivalent chemical properties. Virtual screened compound SCHEMBL16391748 (PubChem CID-117816179) shows the lowest re-rank score. These drugs are identified as high potential IDH2 inhibitors and can halt AML when validated through further In vitro screening.
\end{abstract}

Keywords: Acute myeloid leukemia- IDH2- molecular docking- virtual Screening- pharmacophore-ADMET

Asian Pac J Cancer Prev, 20 (8), 2287-2297

\section{Introduction}

According to the American Cancer Society (ACS), Acute Myeloid Leukemia (AML) is a group of hematological diseases, phenotypic and genetically heterogeneous, characterized by clonal expansion of myeloid with diminished capacity for differentiation. It is characterized by the fast growth of white blood cells, red blood cells, platelets, and it's a build-up in the bone marrow restricting the production of traditional blood cells. Once healthy bone marrow cells are replaced with the leukemic cells, it results in a decline in red blood cells, platelets, and healthy white blood cells. Mainly, symptoms that are seen in patients affected by AML are Weight loss, Fatigue, Fever, Night sweat, Loss of appetite, shortness of breath, natural bruising and bleeding, with lots of body abnormalities and a magnified risk of infection.

According to investigations, 15 to $20 \%$ of acute leukemia cases are in children and $80 \%$ in adults, 3-4 cases of AML per 100,000 adults are diagnosed annually. Among all reported cases, $42.8 \%$ of patients $>65$ years age are rarely diagnosed before the age of 40 years which concludes that Acute Myeloid Leukemia (AML) is predominantly found in adults (Deschler et al., 2006). AML varies with gender; incidence rate of AML is 4.3 and 3 percent per 100,000 males and females respectively and is more commonly found in blacks than whites with the incidence rate of 4.3 and 3.9 respectively per 100,000 (Redaelli et al., 2003).

Since AML is a genetically heterogeneous clonal disorder, the somatic mutation in many genes is identified such as FLT3, C-Kit, N-RAS, NPM1, CEBPA, WT1, ASXL1,

${ }^{1}$ In Silico Research Laboratory, Eminent Biosciences, Vijaynagar, ${ }^{2}$ Bioinformatics Research Laboratory, LeGene Biosciences Pvt Ltd, Mahalakshmi Nagar, Indore, 452010, Madhya Pradesh, ${ }^{3}$ Computer Aided Drug Designing and Molecular Modeling Lab, Department of Bioinformatics, Alagappa University, Karaikudi-630 003, Tamil Nadu, ${ }^{4}$ Department of Biotechnology, Lovely Faculty of Technology and Sciences, Division of Research and Development, Lovely Professional University, Phagwara, Punjab India.*ForCorrespondence: skysanjeev@gmail.com,anuraj@eminentbio.com 
DNMT3A, MLL, IDH1, IDH2 and TET-2 (Marcucci et al., 2011). Recent advances in mutational analysis led to the discovery of isocitrate dehydrogenase $(I D H)$ mutations in AML. $I D H 2$ is an enzyme that catalyzes the oxidative decarboxylation of isocitrate to alpha-ketoglutarate. Its mutated version leads to the accumulation of the oncometabolite (R)-2 hydroxyglutarate, which disrupts several cell processes and leads to a blockage in differentiation. The isocitrate dehydrogenase $I D H 2$ gene is identified to be frequently mutated in acute myeloid leukemia (AML) patients. The $I D H 2$ is located in the mitochondrion and are normally involved in citrate metabolism in the tricarboxylic acid cycle. Targeting IDH2 is compelling, as it is an early and stable mutation in AML (Amaya and Pollyea, 2018). However, hematopoieticstem cell disorder also results in the blockage created by hematopoiesis and overproliferation of immature cells or blast cells which leads to Acute myeloid leukemia (AML) (Shipley et al., 2009). This leukemia gets developed in precursors of myeloid cell lineages due to chromosomal rearrangements and mutation in multiple genes.

Mutation in DNA of stem cells of bone marrow, hematopoietic stem cell, which is responsible for the production of red blood cell, platelets, and white blood cells, causes more production of white blood cells than required. The $I D H 2$ gene also encodes for an enzyme, $\mathrm{NADP}(+)$ dependent homodimer isocitrate dehydrogenase-2, found in mitochondria at chromosome position $15 \mathrm{q} 26.1$ and participates in producing energy for cell activities. The enzyme converts isocitrate compound to alpha-ketoglutarate $(\alpha-K G)$ and produces a molecule NADPH which protects the molecule from highly reactive oxygen species (ROS) (Lu, Venneti et al., 2013). Somatic mutation of the $\mathrm{IDH} 2$ gene was initially identified in $80 \%$ of gliomas and $20 \%$ of acute myeloid leukemia (AMLs) (Lu et al., 2013). However, somatic monoallelic point mutation only manipulates some of the residues consequently. It is not non-functional and it produces 2-hydroxyglutarate (2HG) from alpha-ketoglutarate (Ward et al., 2010).Mutation in the gene was identified by analyzing patients with acute myeloid leukemia (AML) at the position of IDH1R132C, IDH2R140 and IDH2R172K using whole genome sequencing technique. This mutation decreases the affinity of isocitrate dehydrogenase for isocitrate and enhances it for alpha-KG which leads to oxidative decarboxylation of alpha-ketoglutarate to isocitrate. It converts by the reduction of alphaketoglutarate to 2- hydroxyglutarate (D-2HG). Excess accumulation of D-HG causes AML and glioma also. This conversion of the enzyme activity of alpha-KG to 2-DHG, from wild-type to mutant forms the neomorphic activity of enzyme (Ward et al., 2010).

Hence, inhibition of $I D H 2$ plays a vital role in treatment of AML. As the protein does not contain an active catalytic site, blocking $I D H 2$ activation would necessitate an inhibition of dimerization through allosteric interactions. Screening of vast chemical libraries and the use of computational models to evaluate binding ability have revealed a number of compounds that inhibit $I D H 2$ dimerization and exhibit biologic activity against tumor cell. $I D H 2$ represents a promising anticancer target for pharmacologic intervention, due to its central position in numerous signaling pathways.

\section{Materials and Methods}

\section{Selection of Inhibitors}

Pre-existing inhibitors of $I D H 2$ involved in Acute Myeloid Leukemia (AML) were chosen from a few literary works. Four inhibitors were chosen in this study. The inhibitors having PubChem CID, their molecular weight in gm. /mol, H-bond acceptor, H-bond donor and $\log \mathrm{p}$-value is listed in table (Table 1). The 3D structure of each one of those compounds were saved in SDF format. All those inhibitors are indexed accordingly having Pubchem ID with 3D structure (Table 1).

\section{Protein and Ligand preparation}

The crystal structure of target protein, Isocitrate Dehydrogenases (IDH2), was recuperated from Protein Data Bank (PDB ID: 5SVN)and was fetched for further studies of docking process(Xie et al, 2017) (Figure 1). The $3 \mathrm{D}$ conformers of inhibitors having pubchem ID were saved in SDF format. Furthermore, the complete hoard of inhibitors in the form of of 3D structures was prepared using OPLS 2005 force field algorithm embedded in the LegPrep module of Schrödinger suite, 2013 (Schrodinger. LLC, New York, NY) (Babitha et al., 2015; Bandaru et al., 2017a; Basak et al., 2016a, Dunna et al., 2015a). Eventually, in this procedure of preparation, the protein was added with disulfide bonds, missing side chains were filled, water molecules were removed beyond $5 \AA$ from hetero groups, and was saved in the SDF format for further docking studies (Dunna et al., 2015b; Divya et al., 2019; Bandaru et al., 2015a; Kelotra et al., 2014a;Basak et al., 2016b).

\section{Molecular Docking}

The molecular docking studies was performed by using Molegro Virtual Docker (MVD) which is unified with high potential Piece Wise Linear Potential (PLP) and MolDock scoring function (Kelotra et al., 2014b; Bandaru et al., 2014; Khandekar et al., 2016; Bandaru et al., 2013). Subsequently, all cavities were detected to get high volume cavity and hence the highest volume cavity $(>2000 \AA)$ was selected for docking and to target the active of the IDH2 structure (PDB ID: 5SVN) with the pre-prepared 4 ligands. The docking procedure parameters were selected as required, having a maximum population size of 50 , maximum iteration of 1,500 and $0.20 \AA$ as grid resolution (Khandelwal et al., 2018; Bandaru, 2017b; Majhi et al., 2018; Nasr et al., 2015). Further, the post-docking process carries hydrogen bonds optimization and energy minimization, Simplex Evolution at max steps 300 and neighbor distance technical setting fast at 1.00(Sharda $\mathrm{S}$ et al., 2019; Ali MA et al., 2019).

Binding affinity and interactions of ligands with protein was evaluated from the internal electrostatic interaction, internal hydrogen bond interactions, and sp2-sp2 torsions. Later on, the energy of the ligand-receptor complex was minimized using Nelder Mead Simplex Minimization (using non-grid force field and $\mathrm{H}$ bond directionality) 
(Padmini et al., 2019; Sinha et al., 2019; Palak et al., 2019; Patidar et al., 2016). On the basis of the lowest re-ranking score, the best interacting compound was selected for the further studies (Nayarisseri et al., 2018; Bandaru, 2015b; Praseetha et al., 2016; Mendonça-Junior et al., 2019).

\section{Structure-based virtual Screening}

Similarity search was carried out to obtain the best compound having greater affinity other than any established drugs. The filtration properties parameter set by component rule of Lipinski's rule of five at Threshold $>=95 \%$ against NCBI's Pubchem compound database which is a NIH organized public chemical repository of 93 million chemical compounds (Patidar et al., 2019; Monteiro et al., 2019; Rao et al., 2010; Shaheen et al., 2015). As a result, the complete compounds retrieved were again compared with docking studies to obtain a candidate with a better affinity that can halt target protein $\mathrm{IDH} 2$.

\section{Drug - Drug comparative study}

Hereof, the unnamed complex structure of both the outcomes i.e. established compound and virtual screened compound was further imported in Molecular Docker for comparative study. Both the compounds were cleaned by removing constraints. Both these compounds were first exported as the best-posed compounds from the docking results. The comparative result was imported in excel sheet to analyze the affinities, hydrogen interaction, steric energy and high rerank score to identify the best inhibitor.

\section{ADMET studies}

The admetSAR database with ADMET properties of a compound deal with its absorption, distribution, metabolism, excretion, and toxicity in the human body which play the key role in the discovery of a compound. Owing to the superior affinity of the best-established compound and virtual screened compound, the bioactivity properties and toxicity was predicted by using admetSAR (Shameer et al., 2017; Sharda et al., 2017; Sharma et al., 2018; Nayarisseri et al., 2019). The admetSAR database which constitutes the pharmacokinetic profile of a drug molecule is essential in evaluating its pharmacodynamic activities. This database owes free interface interpellation having a distinct biological and chemical contour. The admetSAR database considers on the 5 quantitative regression and 22 qualitative classification models which provide a more established precise result. The free interface of admetSAR tool was used (http://lmmd.ecust. edu.cn:8000/)(Natchimuthu et al., 2016; Chandrakar et al., 2013; Pandey et al., 2013).The compounds which carried greater affinity score from established compounds and virtual screened compounds after docking were used to analyze the ADMET competency (Sinha et al., 2015; Sinha et al., 2014; Trishang et al., 2019; Vuree et al., 2013; Gudala et al., 2015).

\section{Software and web server used}

NCBI PubChem database were used toretrieve the 3D structure of compounds in SDF format to perform virtual screening against the PubChem database. Ligand preparation was optimized by the LegPrep module of
Schrodinger suite 2013. Protein was processed and refined with the protein preparation wizard of Schrodinger suite 2013 (Schrodinger. LLC, 2009, New York, NY). The Flexible molecular docking of the compounds with the target was completed using Molegro Virtual Docker 2010.4.0.0. Accelrys Discovery Studio ${ }^{\circledR}$ Visualizer 3.5.0.12158 (Copyright $^{\odot}$ 2005-12, Accelrys Software Inc.). ADMET profiles were calculated using admetSAR online tool (Laboratory of Molecular Modeling and Design. Copyright ${ }^{@}$ 2012, East China University of Science and Technology, Shanghai Key Laboratory for New Drug Design).

\section{BOILED EGG PLOT}

A Boiled Egg plot, endows a supportive guidance, intuitive, accommodatingly reproducible and an incomparable statistical plot to foreshow the two passive prediction of small molecules, i.e., gastrointestinal absorption and brain-permeant. This model supplies a superior optimization method. Gastrointestinal absorption and Brain access are two crucial pharmacokinetic parameters necessary in estimating the stages of the drug discovery processes. The Brain and IntestinaL EstimateD permeation method (BOILED-Egg) is proposed as an accurate predictive model that works by computing the lipophilicity and polarity of small molecules. This computational model also confers parameters; MW, TPSA, MLOGP, GI and BBB. Herein, if the compound falls on the white ellipse of the plot, the probability of a good intestinal absorption is high. Whereas if the compound falls on the yellow ellipse (i.e. the egg yolk), the probability of Blood-Brain Barrier (BBB) crossing is high. Apart from both the white area and yellow ellipse, the compounds found on the grey area are considered to be out of range of the plot as the molecules are exclusively nonabsorptive by GI and BBB non-permeate. Furthermore, among the established compound the two best drugs based on the lower re-rank score was retrieved and the same procedure with the compounds of the screened compound (Tables 3 and 4).

\section{Results}

Docking results Established Compound Docking Results

The molecular docking studies of 4 pre-established inhibitorswas performed using Molegro Virtual Docker (Table.1). It showed the compound AG-221(PubChem ID-89683805) to be the best established compound among the all pre-established compounds. This compound has the lowest energy with -97.4,976 rerank score and shows the higher affinity score directed towards our target protein IDH2 (Table 2). General properties are: molecular weight $473.383 \mathrm{~g} / \mathrm{mol}, 3$ hydrogen bond donor, 14 hydrogen bond acceptor, topological polar surface area $109 \mathrm{~A}^{2}$ and the $\log \mathrm{P}$ value is 3.5 .

\section{Virtual Screening result}

Further similarity search for AG-221inhibitor resulted in 66 virtual screened compounds (Table 3). The compound with SCHEMBL16391748 (PubChem CID-117816179) has the highest affinity among others. Asian Pacific Journal of Cancer Prevention, Vol 202289 
Table 1. Potential IDH2 Inhibitors with PubChem ID

\begin{tabular}{llcccccc}
\hline S1. & Inhibitors & Pub ID & $\begin{array}{c}\text { MW } \\
(\mathrm{Gm} . / \mathrm{Mol})\end{array}$ & $\begin{array}{c}\text { H-Bond } \\
\text { Acceptor }\end{array}$ & $\begin{array}{c}\text { H-Bond } \\
\text { Donor }\end{array}$ & Log $\mathrm{p}$ & Ref \\
\hline 1 & Enasidenib, AG-221 & 89683805 & 473.383 & 14 & 3 & 3.5 & Sjöblom T et al., 2006 \\
2 & AGI-6780 & 71299339 & 481.508 & 8 & 3 & 4.2 & Sjöblom T et al., 2006 \\
3 & 7-Ketocholesterol & 91474 & 400.647 & 2 & 1 & 7.5 & Katharine Yen et al, 2013 \\
4 & AG-120 & 89699486 & 582.968 & 9 & 1 & 3.4 & (Xiaodong Fu et al., 2014; Stein EM et al., 2016) \\
\hline
\end{tabular}

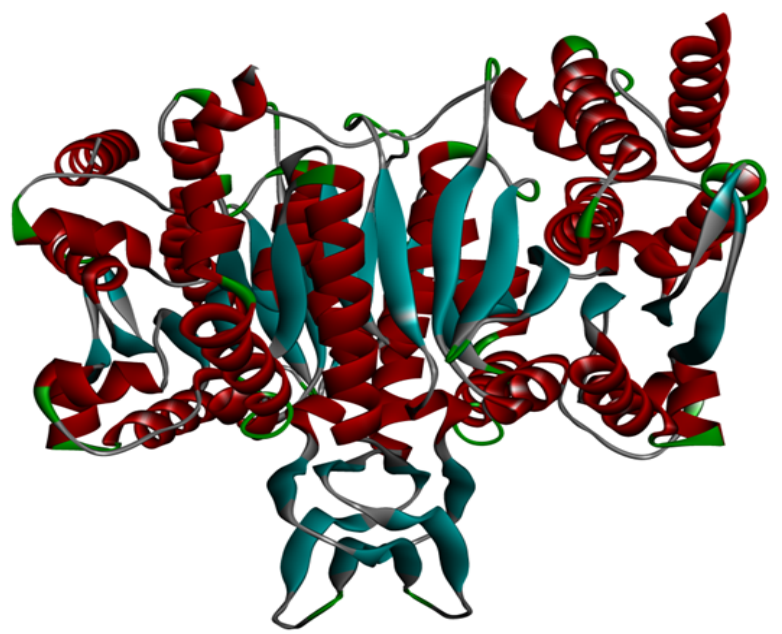

Figure 1. Protein 3D Structure of $I D H-2$ Obtained from PDB (PDBID: 5SVN) Visualization in Accelrys Discovery Studio

This compound has a molecular weight of $468.432 \mathrm{~g} /$ mol 3 hydrogen bond donor and 12 hydrogen bond acceptor, a topological surface area of $95.8 \mathrm{~A}^{2}$ and a $\log \mathrm{P}$ value is 3.7. Among all these 66 virtual screened compounds and 4 pre- established compounds, the drug SCHEMBL16391748 (PubChem CID-117816179) has much potential inhibition against Acute Myeloid Leukemia(AML) over the target protein $I D H 2$.

\section{Pharmacophore studies}

Pharmacophore mapping is a depicting model which provides the finest accuracy of binding site as well as in the discovery of the novel drug. Pharmacophore studies provide accurate query on the optimum interaction with suitable target annotations and represent the aligned poses of the molecule and help us to find the high interaction mode between target protein and compound. This mapping model was employed to identify targets

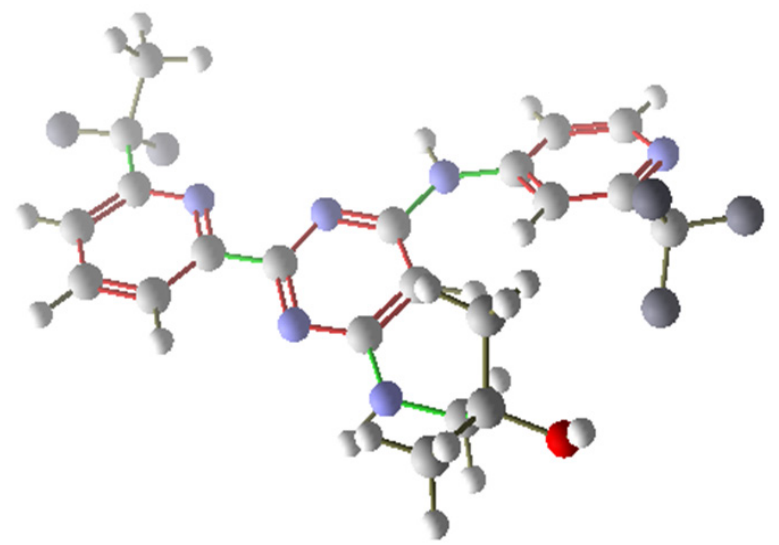

Figure 2. SCHEMBL16391748, PubChem CID: 117816179 Show the Most Effective Compound Binding with $I D H 2$

and the compound interaction in its cavity. Owing to the predicted higher affinity of virtual screened compound SCHEMBL16391748 (PubChem CID: 117816179) with the high-affinity score it was moved ahead for pharmacophore mapping studies. Pharmacophore mapping also results in the positive intensities of electrostatics and there is a variation in intensities of the aromatic compounds and the charges respectively.

The H-bond interactions of the virtual screened compound SCHEMBL16391748 (PubChem CID: 117816179) with lowest rerank score possessing immense affinity at the active sites of $I D H 2$ cavity is shown in (Figure 3). The small green dotted lines are hydrogen bond interaction where SCHEMBL16391748 forms three hydrogen bond interactions with residue Gln316.

(PubChem CID: 117816179) with protein residues involved Gln 316

(Figure 4) shows the residue interaction of virtual screened compound SCHEMBL16391748 PubChem

Table 2.Docking Results for $I D H 2$ Inhibitors

\begin{tabular}{|c|c|c|c|c|c|}
\hline Name & MolDock Score & Rerank Score & Interaction & HBond & MW \\
\hline [02] 89683805 & -131.767 & -97.4976 & -165.709 & -14.7664 & 473.375 \\
\hline [03] 89683805 & -126.856 & -94.9008 & -156.297 & -8.18506 & 473.375 \\
\hline [01] 89699486 & -151.317 & -92.2886 & -140.589 & -9.25299 & 582.961 \\
\hline [01] 89683805 & -127.392 & -91.7372 & -156.855 & -9.9447 & 473.375 \\
\hline [03] 91474 & -114.25 & -91.1626 & -121.211 & -1.92038 & 400.637 \\
\hline [02] 91474 & -115.811 & -91.0259 & -124.627 & -1.51956 & 400.637 \\
\hline [00] 91474 & -117.687 & -90.8601 & -125.468 & -3.84462 & 400.637 \\
\hline [04] 89683805 & -124.328 & -90.6903 & -158.038 & -4.36196 & 473.375 \\
\hline [00] 71299339 & -132.593 & -88.8993 & -146.046 & -6.19172 & 481.511 \\
\hline
\end{tabular}


Table 3.Docking Results for Virtual Screened Compounds

\begin{tabular}{lccccc}
\hline Name & MolDock Score & Rerank Score & Interaction & HBond & MW \\
\hline$[00] 117816179$ & -158.131 & -130.314 & -180.051 & -6.16134 & 468.423 \\
{$[00] 129060692$} & -142.052 & -123.882 & -166.809 & -4.61276 & 419.404 \\
{$[00] 129060700$} & -145.482 & -120.91 & -163.557 & -6.71656 & 419.404 \\
{$[00] 129066642$} & -137.537 & -120.591 & -176.01 & -4.57791 & 491.365 \\
{$[00] 117847478$} & -146.414 & -117.861 & -167.946 & -4.3994 & 473.375 \\
{$[00] 89684023$} & -148.648 & -117.753 & -178.445 & -4.87312 & 473.375 \\
{$[00] 121453832$} & -147.688 & -117.692 & -178.178 & -5 & 473.375 \\
{$[00] 129060704$} & -143.096 & -117.384 & -162.743 & -10.1233 & 419.404 \\
\hline
\end{tabular}

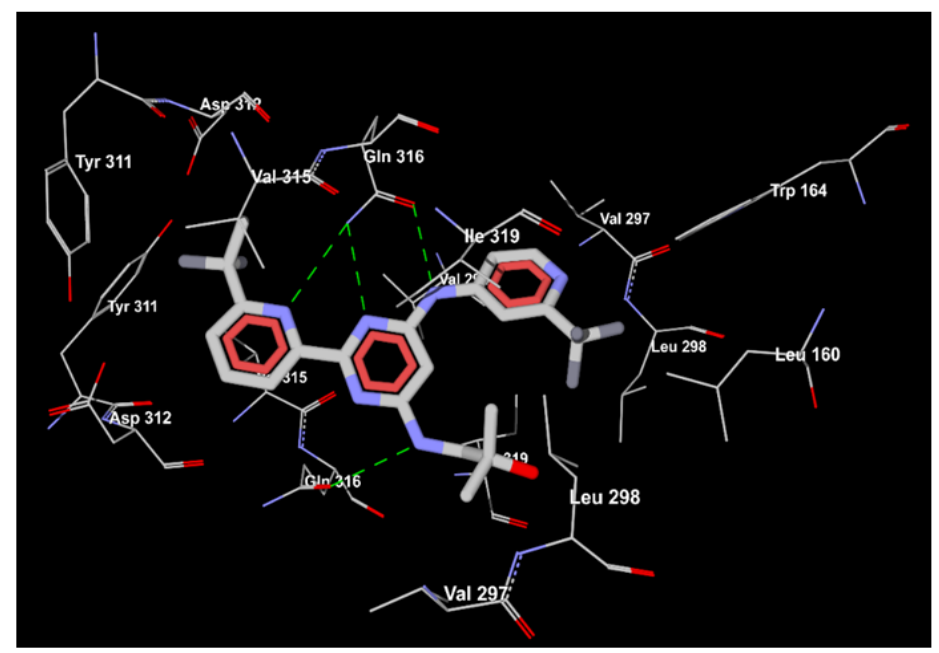

Figure 3. Showing Three H-bond Interaction of Ligand SCHEMBL16391748 (PubChem CID: 117816179) with Protein Residues Involved Gln 316.

CID: 117816179 in the cavity of protein $I D H-2$. The interaction of the residues with ligands which are green circled shows van der Waals interaction and the residues which are pink circled show Electrostatic interactions. A green arrow between the residues and ligands show Hydrogen bond interaction. This figure shows two hydrogen bond interactions with Gln 316 (Figure 4).

Electrostatic interaction of virtual screened compound SCHEMBL 16391748 (PubChem CID: 117816179) with protein $I D H 2$ having high affinity embedded in the protein cavity is shown in (Figure 5). Electrostatic interaction manifests that clusters of charged and polar residues that are detected on protein-protein interfaces and intensify complex stability, although the total effect of electrostatics is generally net destabilizing. The white surface is electrically neutral. The protein carries two types of variant colors for demonstrating positive and negative area, red color revealing electro-negativity zone whereas

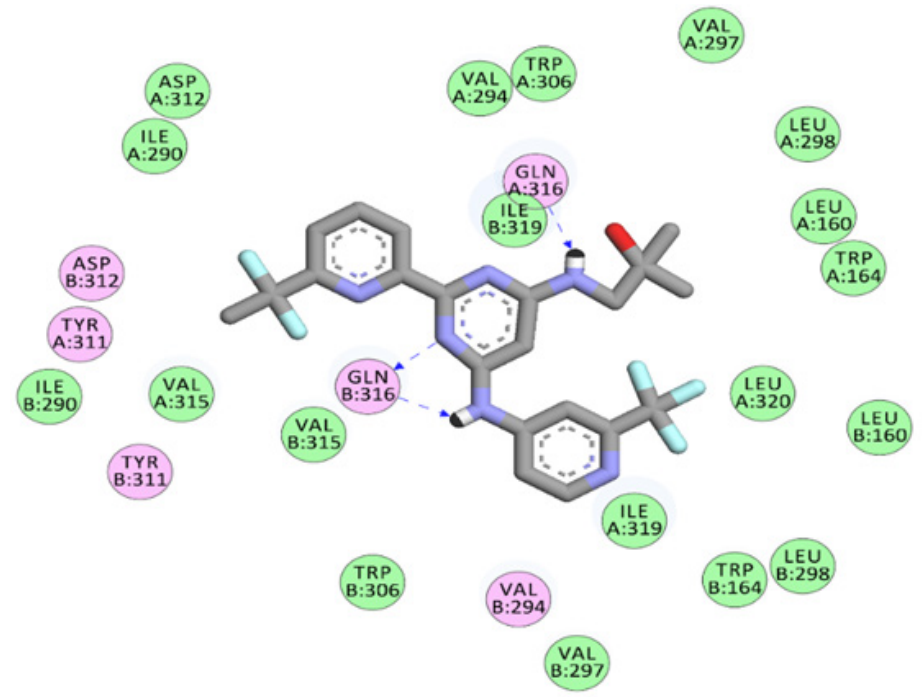

Figure 4. The High Affinity Compound SCHEMBL16391748 (PubChem CID: 117816179) Showing van der Waals Interaction 


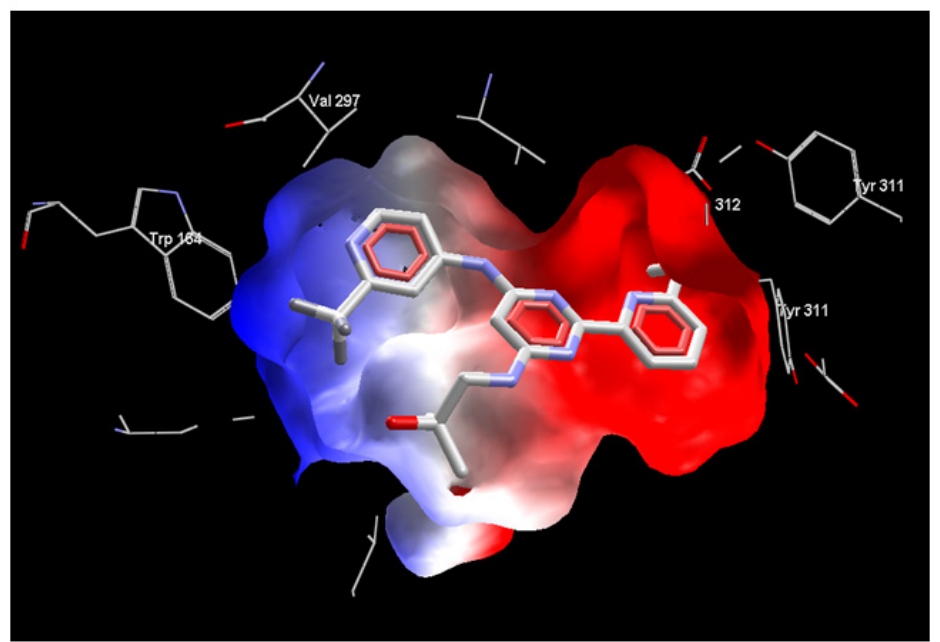

Figure 5. The High Affinity Compound SCHEMBL16391748 (PubChem CID: 117816179) Revealing Electrostatic Interaction

blue color revealing electropositive zone (Figure 5). The target compound SCHEMBL16391748 (PubChem CID: 117816179 ) is surrounded by electronegative residues in red color (Figure 5).

The binding pattern of ligand SCHEMBL16391748 (PubChem CID: 117816179) in the cavity of IDH2 is shown in (Figure 6). The pink lines represent various interactions like electrostatic, van der Waals, stearic, hydrogen bonding and hydrophobic interactions that enable energetically favorable binding of the ligand in the cavity.

The hydrophobic interaction in (Figure 7) imparts an entropic effect and can account for several biophysical events in the protein-ligand binding that are of immense importance in drug design (Figure 7).At the binding site of protein IDH2, virtual screened compound SCHEMBL16391748 CID :(117816179) is interpolated with hydrophobic intensities (Figure 7). As according to figure the hydrophobicity ranges its intensity from 3.00 (maximum hydrophobic zones) to -3.00 (minimum hydrophobic zones). The minimum intensity areas are with blues shaded region and maximum intensity areas are with dull brownish color. Target inhibitor interpolated in dull brown color surfaces show high hydrophobic intensity whereas the pre-established inhibitor AG-120 (PubChem CID-89699486) has very low hydrophobic intensity.

\section{ADMET studies}

The ADMET studies of two compounds i.e. the best pre-established compound AG-221(PubChem ID-89683805) and the virtual screened compound SCHEMBL16391748 (PubChem ID-117816179) revealed slight difference demonstrating equivalent properties (Table 4). The parameter of BBB-Blood-Brain Barrier in ADMET study revealed positive absorption for the candidate drug SCHEMBL16391748 (PubChem ID-117816179). The HIA-Human Intestinal Absorption probability for both the compounds is nearly equal. The values are nearly the samefor P-glycoprotein Substrate and P-glycoprotein Inhibitor for the virtual screened compound. Thereafter, in terms of distribution of subcellular localization in mitochondria, SCHEMBL16391748 (PubChem

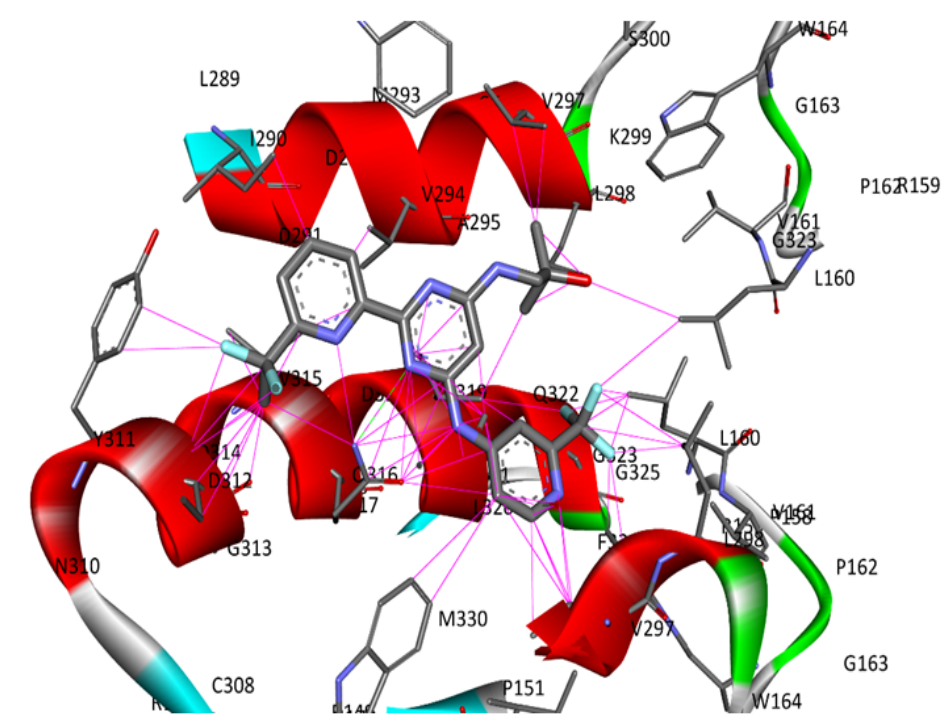

Figure 6. The High Affinity Compound SCHEMBL16391748 (PubChem CID:117816179) Shows Binding Pattern of Ligands 
DOI:10.31557/APJCP.2019.20.8.2287

Identification of High-Affinity Small Molecule Targeting IDH2 for the Clinical Treatment of Acute Myeloid Leukemia Table 4. ADMET Predicted Profile- Classification

\begin{tabular}{|c|c|c|c|}
\hline Model & Result & $\begin{array}{c}\text { Compound AG-221 } \\
\text { CID-:89683805 } \\
\text { Probability }\end{array}$ & $\begin{array}{c}\text { SCHEMBL16391748 } \\
\text { CID-:(117816179) } \\
\text { Probability }\end{array}$ \\
\hline \multicolumn{4}{|l|}{ Absorption } \\
\hline Blood-Brain Barrier & $\mathrm{BBB}+$ & 0.8041 & 0.836 \\
\hline Human Intestinal Absorption & HIA + & 1 & 0.9965 \\
\hline Caco-2 Permeability & Caco2- & 0.5734 & 0.5806 \\
\hline P-glycoprotein Substrate & Substrate & 0.6798 & 0.6585 \\
\hline \multirow[t]{2}{*}{ P-glycoprotein Inhibitor } & Non-inhibitor & 0.6358 & 0.5714 \\
\hline & Non-inhibitor & 0.6694 & 0.525 \\
\hline Renal Organic Cation Transporter & Non-inhibitor & 0.9045 & 0.9283 \\
\hline \multicolumn{4}{|l|}{ Distribution } \\
\hline Subcellular localization & Mitochondria & 0.5694 & 0.5083 \\
\hline \multicolumn{4}{|l|}{ Metabolism } \\
\hline CYP450 2C9 Substrate & Non-substrate & 0.8424 & 0.815 \\
\hline CYP450 2D6 Substrate & Non-substrate & 0.7993 & 0.7935 \\
\hline CYP450 3A4 Substrate & Non-substrate & 0.5651 & 0.5352 \\
\hline CYP450 1A2 Inhibitor & Non-inhibitor & 0.5 & 0.518 \\
\hline CYP450 2C9 Inhibitor & Non-inhibitor & 0.7458 & 0.7473 \\
\hline CYP450 2D6 Inhibitor & Non-inhibitor & 0.7483 & 0.758 \\
\hline CYP450 2C19 Inhibitor & Non-inhibitor & 0.6534 & 0.6195 \\
\hline CYP450 3A4 Inhibitor & Inhibitor & 0.511 & 0.6774 \\
\hline CYP Inhibitory Promiscuity & Low CYP Inhibitory Promiscuity & 0.7468 & 0.5913 \\
\hline \multicolumn{4}{|l|}{ Excretion Toxicity } \\
\hline \multirow[t]{2}{*}{ Human Ether-a-go-go-Related Gene Inhibition } & Weak inhibitor & 0.9772 & 0.9765 \\
\hline & Non-inhibitor & 0.5824 & 0.5645 \\
\hline AMES Toxicity & Non-AMES toxic & 0.7258 & 0.7308 \\
\hline Carcinogens & Non-carcinogens & 0.7461 & 0.7 \\
\hline Fish Toxicity & Low FHMT & 0.5992 & 0.5599 \\
\hline Tetrahymena Pyriformis Toxicity & High TPT & 0.9851 & 0.9889 \\
\hline Honey Bee Toxicity & Low HBT & 0.8319 & 0.8197 \\
\hline Biodegradation & Not ready biodegradable & 1 & 1 \\
\hline Acute Oral Toxicity & III & 0.6553 & 0.6361 \\
\hline Carcinogenicity (Three-class) & Non-required & 0.5086 & 0.4697 \\
\hline
\end{tabular}

ID-117816179) displayed slightly higher probability values with an established best compound. Later on, in terms of metabolism prediction both the compounds show the probability values nearly equal to each other. In case of CYP450 3A4 Inhibitor, virtual screened compound SCHEMBL16391748 (PubChem ID-117816179) has a higher value than the established compound AG-221 (PubChem ID-89683805) whereas in terms of CYP Inhibitory Promiscuity it is the opposite, showing the established compound result having a higher value than the virtual screened compound. Moving forward to excretion and toxicity, both the candidates have equivalent values.

Table 5.ADMET Predicted Profile-Regression

\begin{tabular}{lccc}
\hline Model & Unit & $\begin{array}{c}\text { Compound AG-221 } \\
\text { CID: 89683805 } \\
\text { Value }\end{array}$ & $\begin{array}{c}\text { SCHEMBL16391748 CID-(117816179) } \\
\text { Value }\end{array}$ \\
\hline Absorption & & & \\
$\quad$ Aqueous solubility & LogS & -3.1818 & -3.243 \\
Caco-2 Permeability & LogPapp, cm/s & 0.6503 & 0.6056 \\
Toxicity & & & \\
Rat Acute Toxicity & LD50, mol/kg & 2.5366 & 2.5411 \\
Fish Toxicity & pLC50, mg/L & 1.7437 & 1.6543 \\
Tetrahymena Pyriformis Toxicity & pIGC50, ug/L & 0.3212 & 0.398 \\
\hline
\end{tabular}




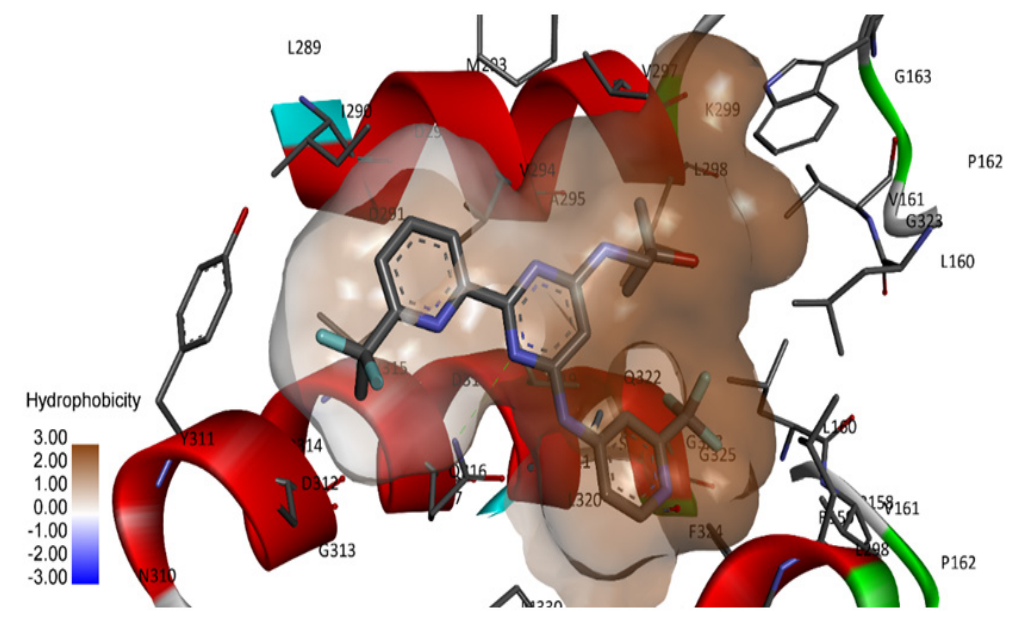

Figure 7. The High Affinity Compound SCHEMBL16391748 (PubChem CID: 117816179) Depicting Hydrophobic Interaction

Furthermore, the ADMET profile prediction on the basis of profile regression and in terms of absorption and toxicity, show that both the compounds are almost equivalent to each other (Table 5).

\section{Drug-Drug comparison studies}

The drug-drug comparative study of two compounds, AG-221 (PubChem ID-89683805) and SCHEMBL16391748 (PubChem ID-117816179) revealed the affinity and interaction respectively (Table 6). The best virtual screened compound SCHEMBL16391748 (PubChem ID-117816179) is represented to have its total energy superior to established docked compound AG-221(PubChem ID-89683805). The steric energy of PLP (Piecewise Linear Potential) and steric energy of LJ12-6 (Leonard-Jones approximation) of these two compound represents that the virtual screened compound SCHEMBL16391748 (PubChem ID-117816179) has

Table 6. Drug-Drug Comparison

\begin{tabular}{|c|c|c|c|c|}
\hline \multirow[b]{2}{*}{ Energy overview: Descriptors } & \multicolumn{2}{|c|}{ Established drug } & \multicolumn{2}{|c|}{ Virtual Screened drug } \\
\hline & MolDock Score & Rerank Score & MolDock Score & Rerank Score \\
\hline Total Energy & -131.764 & -97.495 & -158.118 & -130.317 \\
\hline External Ligand interactions & -165.713 & -131.037 & -180.054 & -149.953 \\
\hline Protein - Ligand interactions & -165.713 & -131.037 & -180.054 & -149.953 \\
\hline Steric (by PLP) & -150.945 & -103.549 & -173.891 & -119.289 \\
\hline Steric (by LJ12-6) & & -15.793 & & -25.783 \\
\hline Hydrogen bonds & -14.768 & -11.696 & -6.163 & -4.881 \\
\hline Hydrogen bonds (no directionality) & & 0 & & 0 \\
\hline Electrostatic (short range) & 0 & 0 & 0 & 0 \\
\hline Electrostatic (long range) & 0 & 0 & 0 & 0 \\
\hline Cofactor - Ligand & 0 & 0 & 0 & 0 \\
\hline Steric (by PLP) & 0 & & 0 & \\
\hline Steric (by LJ12-6) & & 0 & & 0 \\
\hline Hydrogen bonds & 0 & 0 & 0 & 0 \\
\hline Electrostatic & 0 & 0 & 0 & 0 \\
\hline Displaceable Water interactions & 0 & 0 & 0 & 0 \\
\hline Internal Ligand interactions & 33.949 & 33.543 & 21.936 & 19.637 \\
\hline Torsional strain & 14.259 & 13.375 & 1.919 & 1.8 \\
\hline Torsional strain (sp2-sp2) & & 6.979 & & 0.499 \\
\hline Hydrogen bonds & & 0 & & 0 \\
\hline Steric (by PLP) & 22.191 & 3.817 & 20.017 & 3.443 \\
\hline Steric (by LJ12-6) & & 9.372 & & 13.895 \\
\hline Electrostatic & 0 & 0 & 0 & 0 \\
\hline Soft Constraint Penalty & 0 & & 0 & \\
\hline Search Space Penalty & 0 & & 0 & \\
\hline
\end{tabular}




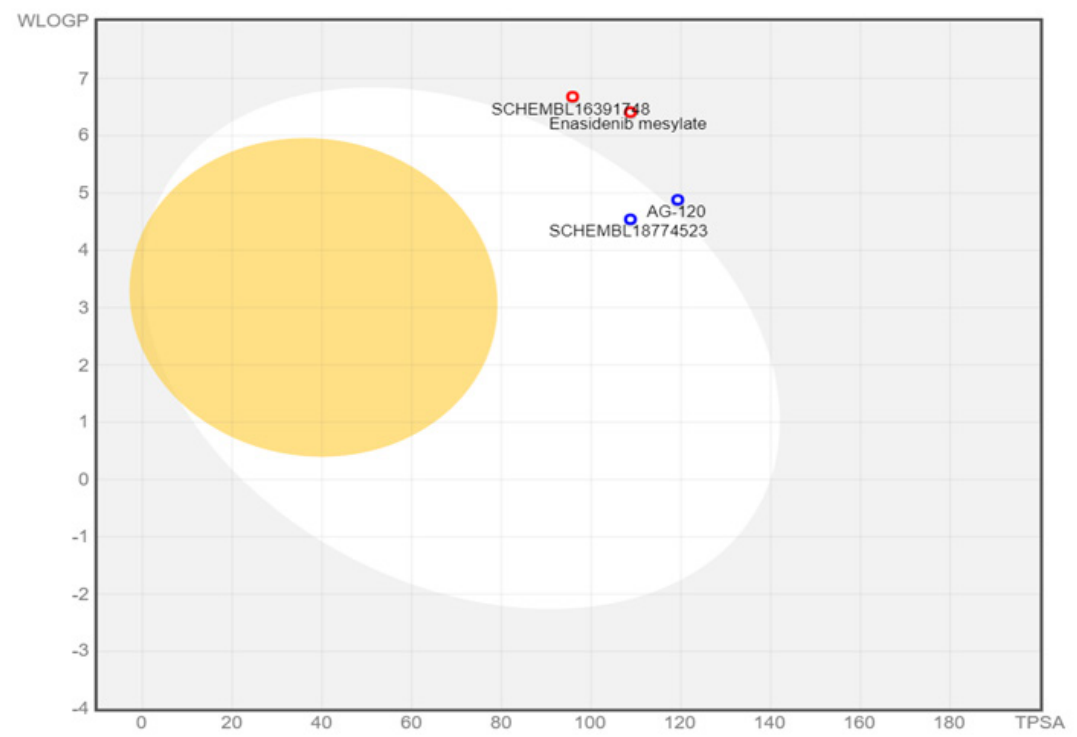

Figure 8. Predictive Model Brain Or IntestinaL EstimateD Permeation Method (BOILED-Egg)

Table 7. Best 6 Compounds from Established Dock Result and Virtual Screened Docked Result Used for Boiled Egg

\begin{tabular}{lccccccc}
\hline Molecule & Pub Id & MW & TPSA & WLOGP & MLOGP & GI absorption & BBB permeant \\
\hline Enasidenib mesylate & 89683805 & 473.37 & 108.74 & 6.41 & 1.41 & Low & No \\
AG-120 & 89699486 & 582.96 & 119.29 & 4.88 & 2.07 & Low & No \\
SCHEMBL16391748 & 117816179 & 468.42 & 95.85 & 6.68 & 2.11 & Low & No \\
SCHEMBL18774523 & 129060692 & 419.4 & 108.74 & 4.54 & 0.68 & High & No \\
\hline
\end{tabular}

high affinity. The hydrogen bond interaction of these two drugs shows high affinity for established drug AG-221(PubChem ID-89683805). As per the complete estimation of comparative study it shows that virtual screened compound SCHEMBL16391748 (PubChem ID117816179 ) has high probability to inhibit protein $I D H 2$.

\section{Boiled Egg Plot Analysis}

The BOILED-Egg plot of 4 candidates having two favorable properties: Blood brain barrier and gastrointestinal absorption bestow an efficient pharmacokinetics profile (Padmini et al., 2019; Sinha et al., 2019; Palak et al., 2019; Patidar et al., 2019; Khandelwal et al., 2018; Majhi et al., 2018). Two compounds were selected from established docked results: Enasidenib mesylate (PubChem ID-89683805) and AG-120(PubChem CID-89699486). Two compounds were selected from virtual docked results: SCHEMBL16391748 (PubChem ID-117816179) and SCHEMBL18774523 (PubChem CID-129060692 (Table 7) SCHEMBL16391748 (PubChem ID-117816179), AG-120(PubChem CID-89699486) and Enasidenib mesylate display low GI-Gastrointestinal absorption and low brain permeation lying under the grey zone of egg plot. AG-120 (PubChem CID-89699486) find its place in the white zone of egg Figure 9, which explains high GI absorption.No compound lie on the yellow ellipse (yolk) and hence are impermeable to the brain. The compound SCHEMBL 18774523 (PubChem CID-129060692) is neither absorbed nor brain penetrable (Table 6).

\section{Discussion}

The study is an attempt to illustrate inhibitors from various literature which inhibits the protein $I D H 2$ for finding new leads in the treatment of acute myeloid leukemia (AML). This study intends to demonstrate inhibitory action2- hydroxyglutarate (D-2HG), by blocking the action of isocitrate dehydrogenase protein. The execution of computational prediction of molecular docking studies shows AG-120 (PubChem CID-89699486) as the best established inhibitor. With reference to this compound, virtual screening and subsequent docking shows a compound SCHEMBL 16391748 (PubChem ID-117816179). Comparative prediction and docking studies for these candidates show that virtual screened docked drug SCHEMBL16391748 has high-affinity score and potential ability to inhibit the activity of IDH2. In this research, we show the pharmacophore mapping of the virtual screened compound SCHEMBL16391748 (PubChem ID-117816179) which depicts optimal binding in the cavity of IDH2 protein with inhibitory ability. Established compound AG-120 and virtual screened compound SCHEMBL16391748 (PubChem ID-117816179) were examined for ADMET study which shows the compounds as non-carcinogenic and non-mutagenic having analogous properties. According to the results, the virtual screened compound was found having a high probability of HIA-Human Intestinal Absorption and high BBB-Blood Brain Barrier probability besides showing non AMES toxicity. Prediction with BOILED-Egg plot shows 
that SCHEMBL16391748 (PubChem ID-117816179) represents high bioavailable optimization as compared to other drugs: PubChem CID-129060692, pre-established compound AG-120(PubChem CID-89699486) and Enasidenib mesylate(PubChem ID-89683805). Virtually screened inhibitor SCHEMBL16391748 (PubChem ID-117816179) also showed a very low rerank score describing its stability with the protein. On consideration of all the above properties, the virtual screened compound SCHEMBL16391748 (PubChem ID-117816179) can be a potential inhibitor for the protein and can emerge as an important drug in the treatment of AML in future ahead.

\section{Acknowledgements}

SKS thank Department of Biotechnology (DBT), New Delhi (No. BT/PR8138/BID/7/458/2013, dated 23rd May, 2013), DST-PURSE 2nd Phase Programme Order No. SR/PURSE Phase 2/38 (G dated 21.02.2017 and FIST (SR/FST/LSI - 667/2016), MHRD RUSA - Phase 2.0 (grant sanctioned vide letter No. F. 24-51 / 2014 - U, Policy (TNMulti-Gen), Dept. of Edn. Govt. of India, Dt. 09.10.2018) for providing the financial assistance.

\section{Author contributions}

All authors have been personally and actively involved in substantive work leading to the manuscript, and will hold themselves jointly and individually responsible for its content.

\section{Compliance with ethical standards \\ Conflict of interest}

The authors declare that they have no conflict of interest.

\section{Ethical approval}

Ethical approval and informed consent not required for this manuscript.

\section{References}

Ali, M A, Vuree S, Goud H, et al (2019). Identification of highaffinity small molecules targeting gamma secretase for the treatment of alzheimer's disease. Curr Top Med Chem, 19, 1173-87.

Amaya ML, Pollyea DA, (2018). Targeting the IDH2 pathway in acute myeloid leukemia. Clin Cancer Res, 24, 4931-6.

Babitha PP, Sahila MM, Bandaru S, et al (2015). Molecular docking and pharmacological investigations of RivastigmineFluoxetine and Coumarin-Tacrine hybrids against Acetyl Choline Esterase. Bioinformation, 11, 378-86.

Bandaru S, Alvala M, Nayarisseri A, et al (2017a). Molecular dynamic simulations reveal suboptimal binding of salbutamol in T164I variant of beta2 adrenergic receptor. PLoS One, 12, e0186666.

Bandaru S, Marri VK, Kasera P, et al (2014). Structure based virtual screening of ligands to identify cysteinyl leukotriene receptor 1 antagonist. Bioinformation, 10, 652-7.

Bandaru S, Ponnala D, Lakkaraju C, et al (2015a). Identification of high affinity non-peptidic small molecule inhibitors of MDM2-p53 interactions through structure-based virtual screening strategies. Asian Pac J Cancer Prev, 16, 3759-65.

Bandaru S, Prasad MH, Jyothy A, et al (2013). Binding modes and pharmacophoric features of muscarinic antagonism and beta2 agonism (MABA) conjugates. Curr Top Med Chem, 13, 1650-5.

Bandaru S, Sumithnath TG, Sharda S, et al (2017b). Helix-Coil transition signatures $B$-Raf V600E mutation and virtual screening for inhibitors directed against mutant $B$-Raf. Curr Drug Metab, 18, 527-34.

Bandaru S, Tiwari G, Akka J, et al (2015b). Identification of high affinity bioactive Salbutamol conformer directed against mutated (Thr164Ile) beta 2 adrenergic receptor. Curr Top Med Chem, 15, 50-6.

Basak SC, Nayarisseri A, Gonzalez-Diaz H, et al (2016a). Editorial (Thematic Issue: Chemoinformatics Models for Pharmaceutical Design, Part 1). Curr Pharm Des, 22, 5041-2.

Basak SC, Nayarisseri A, Gonzalez-Diaz H, et al (2016b). Editorial (Thematic Issue: Chemoinformatics Models for Pharmaceutical Design, Part 2). Curr Pharm Des, 22, 5177-8.

Chandrakar B, Jain A, Roy S, et al (2013). Molecular modeling of Acetyl-CoA carboxylase (ACC) from Jatropha curcas and virtual screening for identification of inhibitors. $J$ Pharm Res, 6, 913-8.

Deschler B, Lübbert M, et al (2006). Acute myeloid leukemia: epidemiology and etiology. Cancer, 107, 2099-2107.

Divya Jain TU, Shreshtha S, Aishwarya G, et al (2019). Design of novel JAK3 inhibitors towards Rheumatoid Arthritis using molecular docking analysis. Bioinformation, 15, 68-78.

Dunna NR, Bandaru S, Raj Akare U, et al (2015a). Multiclass comparative virtual screening to identify novel Hsp90 inhibitors: a therapeutic breast cancer drug target. Curr Top Med Chem, 15, 57-64.

Dunna NR, Kandula V, Girdhar A, et al (2015b). High affinity pharmacological profiling of dual inhibitors targeting RET and VEGFR2 in inhibition of kinase and angiogeneis events in medullary thyroid carcinoma. Asian Pac J Cancer Prev, 16, 7089-95.

Fu X, Huang X, Li P, et al (2014). 7-Ketocholesterol inhibits isocitrate dehydrogenase 2 expression and impairs endothelial function via microRNA-144. Free Radical Bio Med, 71, 1-15

Gudala S, Khan U, Kanungo N, et al (2015). Identification and pharmacological analysis of high efficacy small molecule inhibitors of EGF-EGFR interactions in clinical treatment of non-small cell lung carcinoma: A computational approach. Asian Pac J Cancer Prev, 16, 8191-6.

Kelotra A, Gokhale SM, Kelotra S, et al (2014a). Alkyloxy carbonyl modified hexapeptides as a high affinity compounds for Wnt5A protein in the treatment of psoriasis. Bioinformation, 10, 743-9.

Kelotra S, Jain M, Kelotra A, et al (2014b). An in silico appraisal to identify high affinity anti-apoptotic synthetic tetrapeptide inhibitors targeting the mammalian caspase 3 enzyme. Asian Pac J Cancer Prev, 15, 10137-42.

Khandekar N, Singh S, Shukla R, et al (2016). Structural basis for the in vitro known acyl-depsipeptide 2 (ADEP2) inhibition to Clp 2 protease from Mycobacterium tuberculosis. Bioinformation, 12, 92-7.

Khandelwal R, Chauhan A, Hussain T, et al (2018). Structure-based virtual screening for the identification of high affinity small molecule towards STAT3 for the clinical treatment of Osteosarcoma. Curr Top Med Chem, 18, 2511-26.

Lu C, Sriram V, Altuna A, et al (2013). Induction of sarcomas by mutant IDH2. Genes Dev, 27, 1986-8.

Majhi M, Ali MA, Limaye A, et al (2018). An in silico investigation of potential EGFR inhibitors for the clinical 
treatment of colorectal cancer. Curr Top Med Chem, 18, 2355-66.

Marcucci G, Haferlach T, Döhner H, et al (2011). Molecular genetics of adult acute myeloid leukemia: prognostic and therapeutic implications. J Clin Oncol, 29, 475-86.

Mardis ER, Ding L, Dooling DJ, et al (2009). Recurring mutations found by sequencing an acute myeloid leukemia genome. $N$ Engl J Med, 361, 1058-66

Mendonça-Junior FJ, Scotti MT, Nayarisseri A, et al (2019). Natural bioactive products with antioxidant properties useful in neurodegenerative diseases. Oxid Med Cell Longev, 2019, 1-2.

Monteiro AFM, Viana JDO, Nayarisseri A, et al (2018). Computational studies applied to flavonoids against Alzheimer's and Parkinson's diseases. Oxid Med Cell Longev, 2018, 1-21.

Nasr AB, Ponnala D, Sagurthi SR, et al (2015). Molecular Docking studies of FKBP12-mTOR inhibitors using binding predictions. Bioinformation, 11, 307-15.

Natchimuthu V, Bandaru S, Nayarisseri A, et al (2016). Design, synthesis and computational evaluation of a novel intermediate salt of N-cyclohexyl-N-(cyclohexylcarbamoyl)4-(trifluoromethyl) benzamide as potential potassium channel blocker in epileptic paroxysmal seizures. Comput Biol Chem, 64, 64-73.

Nayarisseri A, Singh SK.(2019). Functional inhibition of VEGF and EGFR suppressors in cancer treatment. Curr Top Med Chem, 19, 178-9.

Nayarisseri A, Hood EA, ???, et al (2018). Advancement in Microbial Cheminformatics. Curr Top Med Chem, 18, 2459-61.

Padmini G, Aashish PSC, Anushka A, et al (2019). FLT3 inhibitor design using molecular docking based virtual screening for acute myeloid leukemia. Bioinformation, 15, 104-15.

Palak S, Ravina K, Diksha S, et al (2019). Virtual screening of IL-6 inhibitors for idiopathic Arthritis. Bioinformation, 15, 121-30.

Pandey N, Yadav M, Nayarisseri A, et al (2013). Cross evaluation of different classes of alpha-adrenergic receptor antagonists to identify overlapping pharmacophoric requirements. J Pharm Res, 6, 173-8.

Patidar K, Deshmukh A, Bandaru S, et al (2016). Virtual screening approaches in identification of bioactive compounds Akin to Delphinidin as potential HER2 inhibitors for the treatment of breast cancer. Asian Pac J Cancer Prev, 17, 2291-5.

Patidar K, Panwar U, Vuree S, et al (2019). An in silico approach to identify high affinity small molecule targeting m-TOR inhibitors for the clinical treatment of breast cancer. Asian Pac J Cancer Prev, 20, 1229-41.

Praseetha S, Bandaru S, Nayarisseri A, et al (2016). Pharmacological analysis of Vorinostat analogues as potential anti-tumor agents targeting human histone deacetylases: an Epigenetic treatment stratagem for cancers. Asian Pac J Cancer Prev, 17, 1571-6.

Rao DM, Nayarisseri A, Yadav M, et al (2010). Comparative modeling of methylentetrahydrofolate reductase (MTHFR) enzyme and its mutational assessment: in silico approach. Int J Bioinformatics Res, 2, 5-9.

Redaelli A, Lee JM, Stephens JM, et al (2003). Pashos, C. L. Epidemiology and clinical burden of acute myeloid leukemia. Expert Rev Anticancer Ther, 3, 695-710.

Sharda S, Khandelwal R, Adhikary R, Sharma D, Majhi M, Hussain T, et al (2019). A computer-aided drug designing for pharmacological inhibition of ALK inhibitors induces apoptosis and differentiation in Non-small cell lung cancer.
Curr Top Med Chem, 19, 1129-44.

Shaheen U, Akka J, Hinore JS, et al (2015). Computer aided identification of sodium channel blockers in the clinical treatment of epilepsy using molecular docking tools. Bioinformation, 11, 131-7.

Shameer K, Nayarisseri A, Duran FXR, et al (2017). Improving neuropharmacology using big data, machine learning and computational algorithms. Curr Neuropharmacol, 15, 1058-61.

Sharda S, Sarmandal P, Cherukommu S, et al (2017). A virtual screening approach for the identification of high affinity small molecules targeting BCR-ABL1 inhibitors for the treatment of chronic myeloid leukemia. Curr Top Med Chem, 17, 2989-96.

Sharma K, Patidar K, Ali MA, et al (2018). Structure-based virtual screening for the identification of high affinity compounds as potent VEGFR2 inhibitors for the treatment of renal cell carcinoma. Curr Top Med Chem, 18, 2174-85.

Shen Y, Zhu YM, Fan X, et al (2011). Gene mutation patterns and their prognostic impact in a cohort of 1,185 patients with acute myeloid leukemia. Blood, 118, 5593-5603.

Shipley JL, Butera JN, ???, et al (2009). Acute myelogenous leukemia. Exp Hematol, 37, 649-58.

Sinha C, Nischal A, Bandaru S, et al (2015). An in silico approach for identification of novel inhibitors as a potential therapeutics targeting HIV-1 viral infectivity factor. Curr Top Med Chem, 15, 65-72.

Sinha C, Nischal A, Pant KK, et al (2014). Molecular docking analysis of RN18 and VEC5 in A3G-Vif inhibition. Bioinformation, 10, 611-6.

Sinha K, Majhi M, Thakur G, et al (2018). Computer aided Drug Designing for the identification of high affinity small molecule targeting CD20 for the clinical treatment of Chronic Lymphocytic Leukemia (CLL). Curr Top Med Chem, 18, 2527-42.

Sjöblom T, Jones S, Wood LD, et al (2006). The consensus coding sequences of human breast and colorectal cancers. Science, 314, 268-274.

Stein EM, Tallman MS, et al (2016). Emerging therapeutic drugs for AML. Blood, 127, 71-78.

Trishang U, Sourav M, Khushboo S, et al (2019). Design of PD-L1 inhibitors for lung cancer. Bioinformation, 15, 139-50.

Vuree S, Dunna NR, Khan IA, et al (2013). Pharmacogenomics of drug resistance in Breast Cancer Resistance Protein (BCRP) and its mutated variants. J Pharm Res, 6, 791-8.

Wang F, Travins J, Chen Y, et al (2013). AG-221 offers a survival advantage in a primary human $I D H 2$ mutant AML xenograft model. Blood, 122, 240.

Ward PS, Patel J, Wise DR, et al (2010). The common feature of leukemia-associated IDH1 and IDH2 mutations are a neomorphic enzyme activity converting $\alpha$-ketoglutarate to 2-hydroxyglutarate. Cancer Cell, 17, 225-34.

Xie X, Baird D, Bowen K, et al (2017). Allosteric mutant IDHI inhibitors reveal mechanisms for IDH1 mutant and isoform selectivity. Structure, 25, 506-13.

This work is licensed under a Creative Commons AttributionNon Commercial 4.0 International License. 